\title{
Confocal Microscopy and its Role in Teledermatology: Diagnosis of Basal Cell Carcinoma in a Clinical Setting
}

\author{
Jason Garfinkel ${ }^{1}$; C Chandler ${ }^{1}$; G Rubinstein ${ }^{1}$; Manu Jain ${ }^{2}$ \\ ${ }^{1}$ Dermatology and Laser Centre of Studio City, Los Angeles, CA, United States \\ ${ }^{2}$ Department of Dermatology, Memorial Sloan-Kettering Cancer Center, New York, NY, United States
}

\section{Corresponding Author:}

Manu Jain

Department of Dermatology

Memorial Sloan-Kettering Cancer Center

530 E 74th St

New York, NY, 10021

United States

Phone: 16466083562

Email: jainm@mskcc.org

\section{Abstract}

Background: Reflectance confocal microscopy (RCM) is a noninvasive tool that is used to diagnose skin cancers. However, RCM requires an expert consultation, which is often performed via store-and-forward (SAF) teledermatology. Unfortunately, SAF does not mimic bedside diagnosis, nor permits interaction between the remote expert reader, physician, and patient. Recently, a live interactive method (LIM)-tele-RCM approach was shown to diagnose basal cell carcinoma (BCC) from a remote location, demonstrating advantages over SAF by providing a bedside diagnosis during consultation.

Objective: The aim of this study is to validate the LIM-tele-RCM approach to diagnose BCC in a real-world setting.

Methods: In this pilot study, 4 patients with 6 clinically suspicious BCC lesions were enrolled and imaged with RCM at a Los Angeles dermatology clinic. A Health Insurance Portability and Accountability Act-compliant teleconferencing application was used to livestream RCM images to an expert RCM reader in New York. The expert reader had remote control of the software, direct audio communication with the clinic, and the patient's clinical history with dermoscopy. During imaging, RCM features were noted, and a diagnosis was made at the bedside. After imaging, patients completed a short questionnaire (on a 5-point scale, with 5 being the highest score) about satisfaction, comfort, and communication during the session.

Results: RCM diagnosed 4/6 (67\%) lesions correctly as BCC and 2/6 (33\%) were false-positive diagnoses. The true-positive lesions had "tumor islands with palisading and clefting" and were directly managed with Mohs surgery. The false-positive lesions had "dark silhouettes" (a common false-positive feature for BCC) and underwent a shave biopsy for confirmation. The entire session ranged from 15 to 20 minutes (an average of 17.7 minutes), comparable to the reported RCM procedure time. On the questionnaire, all patients responded with the highest rating $(5 / 5)$ for each question.

Conclusions: LIM-tele-RCM demonstrates potential advantages over the SAF method, enabling bedside diagnosis with similar diagnostic accuracy as reported in the literature and proper management. Additionally, the remote reader had access to patients' clinical backgrounds and could engage with patients. It may also be useful for training novice RCM users and beneficial in settings where remote diagnostics are desired, such as during the COVID-19 pandemic. However, technical challenges such as image quality degradation during video streaming, poor internet bandwidth, and end user latency may impact diagnosis. Larger, multicenter studies are needed to assess the accuracy of LIM-tele-RCM for the diagnosis of BCC and other neoplastic and inflammatory lesions, and to quantify technical limitations.

Acknowledgments: This work was funded by the National Cancer Institute Cancer Center (Grant P30 CA008748).

Conflicts of Interest: None declared.

(iproc 2022;8(1):e36906) doi: $10.2196 / 36906$

\section{KEYWORDS}

reflectance confocal microscopy; teledermatology; telemedicine; tele-RCM; skin cancer; basal cell carcinoma 


\section{Multimedia Appendix 1}

Basal cell carcinoma (BCC) diagnosed remotely using the LIM-tele-RCM method, dermoscopy, and histopathology confirmation. (a) Screenshot showing the remote expert RCM reader controlling RCM image acquisition software via the teleconferencing and screensharing application (blue arrow) to diagnose $\mathrm{BCC}$, and with direct audio communication to the patient and clinic (yellow arrow). Real-time, zoomed-in RCM image navigation during the imaging session allowed the remote expert to identify BCC foci based on the recognition of "tumor islands with palisading and clefting" (yellow asterisk). (b) Dermoscopy shows pink background with telangiectatic vessels and blue-grey globules (yellow arrow). (c) The lesion was biopsied, confirming the diagnosis of a nodular BCC (yellow asterisk) on a hematoxylin and eosin-stained tissue section (10x).

[PNG File, $241 \mathrm{~KB}-$ Multimedia Appendix 1]

\section{Multimedia Appendix 2}

Patient demographics, lesion characteristics, reflectance confocal microscopy (RCM) features, RCM diagnosis, histopathology diagnosis, and time of imaging.

[DOCX File, 15 KB-Multimedia Appendix 2]

Edited by T Derrick; this is a non-peer-reviewed article. Submitted 28.01.22; accepted 28.01.22; published 02.03.22.

Please cite as:

Garfinkel J, Chandler C, Rubinstein G, Jain M

Confocal Microscopy and its Role in Teledermatology: Diagnosis of Basal Cell Carcinoma in a Clinical Setting

iproc 2022;8(1):e36906

URL: https://www.iproc.org/2022/1/e36906

doi: $10.2196 / 36906$

PMID:

CJason Garfinkel, C Chandler, G Rubinstein, Manu Jain. Originally published in Iproceedings (https://www.iproc.org), 02.03.2022. This is an open-access article distributed under the terms of the Creative Commons Attribution License (https://creativecommons.org/licenses/by/4.0/), which permits unrestricted use, distribution, and reproduction in any medium, provided the original work, first published in Iproceedings, is properly cited. The complete bibliographic information, a link to the original publication on https://www.iproc.org/, as well as this copyright and license information must be included. 